\title{
X-ray emission study of extreme blazars using AstroSat
}

\author{
Pranjupriya Goswami, ${ }^{a, b, *}$ Atreyee Sinha, ${ }^{c}$ Nijil Mankuzhiyil, ${ }^{d}$ Ranjeev Misra, ${ }^{e}$ \\ Zahoor Malik, ${ }^{f}$ Sunder Sahayanathan, ${ }^{d}$ Sunil Chandra ${ }^{a, g}$ and Rupjyoti Gogoi ${ }^{b}$ \\ ${ }^{a}$ Center for Space Research, North-West University, Potchefstroom, 2520, South Africa \\ ${ }^{b}$ Department of Physics, Tezpur University, Napaam - 784028, India \\ ${ }^{c}$ Laboratoire Univers et Particules de Montpellier, Université de Montpellier, CNRS 34095, France \\ ${ }^{d}$ Astrophysical Sciences Division, Bhabha Atomic Research Centre, Mumbai - 400085, India \\ ${ }^{e}$ Inter-University Center for Astronomy and Astrophysics, Post Bag 4, Ganeshkhind, Pune - 411007, India \\ ${ }^{f}$ Department Of Physics, University of Kashmir, Srinagar-190006, India \\ ${ }^{g}$ South African Astronomical Observatories, Observatory, Cape Town 7925, South Africa \\ E-mail: pranjupriya.g@gmail.com
}

The X-ray emission in high energy peaked blazars exhibit curved spectral nature and is interpreted in terms of a log-parabola electron distribution, or due to an energy-dependent electron diffusion from the acceleration regions. This unique feature may uncover relevant information about the acceleration mechanisms and the underlying physical conditions in the jet. However, the X-ray emission properties in the extreme class of blazars (termed as "EHBLs") are poorly understood due to insufficient data at hard X-ray energies. The broadband coverage blazar monitoring programme of the AstroSat, covering UV to X-ray energy range, has given us unprecedented simultaneous X-ray data from the SXT (0.3-8 keV) and the LAXPC (3-80 keV) instruments. This wide range $\mathrm{X}$-ray data can be used to constrain several model parameters such as synchrotron peak energy, spectral index and the high energy cut off in given electron distribution. In this contribution, we report on a detailed spectral study of the HBL sources 1ES 1741+196 and 1ES 2322-409 using data from AstroSat. The observations of 1ES 1741+196 and 1ES 2322-409 were made as part of AstroSat AO proposals, observed during 2019 and 2020 respectively. We investigate their $\mathrm{X}$-ray spectral nature through spectral fitting using different curved spectral models and provide a well-constrained measure of the synchrotron peak energies.

$3^{\text {th }}$ International Cosmic Ray Conference (ICRC 2021)

July 12th - 23rd, 2021

Online - Berlin, Germany

\footnotetext{
${ }^{*}$ Presenter
} 


\section{Introduction}

Blazars are a class of active galactic nuclei (AGNs) with a relativistic jet oriented close to the line of the sight of the observer. The observed emission is non-thermal and doppler boosted in nature $[1,2]$. They are further characterized by rapid variable continuum emission at varying time scales and across the entire electromagnetic (EM) spectrum. The spectral energy distributions (SEDs) of these objects appear as double peaked with one peak at low energy in the range from IR to X-ray band and the other peak at $\gamma$-ray energies in $\mathrm{GeV} / \mathrm{TeV}$ [4]. The low energy SED component is well understood to be arising due to synchrotron emission from highly relativistic electrons (or protons) gyrating in the magnetic field of the jet [3]. The synchrotron peak in high energy peaked blazars (HBLs) falls in an energy range from tens of $\mathrm{eV}$ to a few $\mathrm{keV}$. Interestingly, the X-ray spectrum is known to exhibit a curvature that deviates considerably from a conventional power-law form. While the traditional explanation for the curved X-ray spectrum was attributed due to a synchrotron cooling break, well resolved spectral measurements now reveal that a broken power law does not well explain the break. An analytic log-parabola model provides a better representation [5, 6], but provides little understanding of the underlying physics. An alternative description for attaining this curvature from synchrotron emission is the energy-dependent electron diffusion (EDD model, [7, 8]). Further, EDD model connects the curvature parameter with the magnetohydrodynamic (MHD) turbulent index and the turbulence type, and thereby, providing more details about the underlying physical processes.

An extreme class of HBLs (or EHBLs), on the other hand, exhibit unique spectral behaviour with hard X-ray and TeV spectra where their SED peaks are located at high energies in these two energy bands [9]. These extreme properties are difficult to interpret within the standard acceleration/emission theories. As for the X-ray emission, the synchrotron peak energies are not constrained for a number of such sources due to the lack of data in hard X-rays. The SED peak energies, however, are an important factor that would allow us to constrain the particle spectrum and intrinsic source parameters. A detailed spectral study of these extreme sources may provide new insight into the underlying physical conditions, however, this necessitates simultaneous MWL data, especially wide coverage in X-rays and VHE.

In this work, we investigate the X-ray emission features of the HBL 1ES 1741+196 and 1ES 2322-409. The X-ray spectral properties and variability of these sources are unclear and also, no sufficient data is available in this energy band. The HBL source 1ES 1741+196 is suspected to be an EHBL type, however, this result is inconclusive due to the lack of hard X-ray data. Therefore, the wide range AstroSat data reported here can make a crucial contribution to the understanding of the hard X-ray spectrum of this source. For spectral analysis, we use various synchrotron curved spectral models developed under different descriptions of particle acceleration and diffusion. We then discuss the preliminary spectral analysis results at different flux states and provide the possible interpretation to the X-ray spectral curvature of the source spectra.

1ES 1741+196 is an HBL, located at coordinates (J2000) $\alpha=17: 43: 57.8$ (hh:mm:ss), $\delta=19: 35: 09$ (dd:mm:ss) and redshift of $z=0.084$ [10]. The source has been detected in almost all the wavelengths from radio to very high energy (VHE) $\gamma$-rays $[11,12,15]$. The broadband SED of 1ES 1741+196 has been studied by both MAGIC and VERITAS lead campaigns [13, 14], supplemented with contemporaneous multi-instrument data from Swift and Fermi-LAT. The authors explained the 
non-thermal emission under the framework of one-zone SSC models, however, there were a few caveats mainly at the optical and X-ray bands. In the case of the X-ray band, it is interesting to note a harder spectrum, indicating the synchrotron peak energy may be located at higher X-ray energy above $\sim 5 \mathrm{keV}$. Nevertheless, having a well-constrained IC peak from the combined Fermi-LAT and MAGIC/VERITAS observations, the lack of hard X-ray data and an unconstrained synchrotron peak may lead to large uncertainties in the emission parameters. For this very reason, it is worthwhile to investigate the nature of the X-ray spectrum, especially beyond XRT observational energy range.

1ES 2322-409 (RA 23:24:48 (hh:mm:ss), DEC -40:39:36 (dd:mm:ss)) is classified as an HBL, while the redshift is unknown. Although the source has been observed in a wide range of energies, it is not well studied and the spectral nature is poorly understood. In the recent study made by Abdalla et al. (2019) [16], the authors have modelled the VHE observations with quasi-simultaneous data from various waveband instruments. The broadband study includes the different pointing of X-ray observations from Swift-XRT and RXTE-PCA, $\gamma$-ray observations from Fermi-LAT. The multiwavelength SED corresponding to the highest state XRT flux with contemporaneous observations is modelled for two different values of redshift, $z=0.174$ and $z=0.06$ (test value) using a broken power-law (BPL) with a high energy break. The XRT spectrum showed the hardest power-law index as $\Gamma \sim 2.14$ and no indication of spectral curvature. Whereas its synchrotron peak was predicted at an energy $\sim 0.04 \mathrm{keV}$. Nevertheless, The different epoch X-ray and optical/UV spectra from Swift showed variations in their spectral indices, and such variations may provide crucial information about the particle acceleration mechanisms.

\section{AstroSat observations and data analysis}

AstroSat is a multi-wavelength space observatory that consists of five instruments on board covering a wide range of energies from UV to hard X-ray up to $150 \mathrm{keV}$, launched in September 2015 from ISRO, India. The instruments onboard AstroSat are: Soft X-ray focusing Telescope (SXT) operates in the energy range 0.3-8 keV, Large Area X-ray Proportional Counters (LAXPC) in 3-80 keV, UltraViolet Imaging Telescope (UVIT) with five filters of each NUV \& FUV channels, Cadmium Zinc Telluride Imager (CZTI) in 10-100 keV and Scanning Sky Monitor (SSM). A detailed description of AstroSat is provided in Agrawal (2006) [17] and Singh et al. (2014) [18]. We had proposed the AstroSat observation of HBLs 1ES 1741+196 and 1ES 2322-409 during 2018 \& 2020 (AO cycle $05 \&$ 09), with SXT as a primary instrument and also configured for LAXPC and UVIT instruments for simultaneous observations. 1ES 1741+196 was observed for 3-pointing during 2019 and this data is publicly available in AstroSat archive. 1ES 2322-409 was observed for single pointing during July 2020, and the data is within the proprietary period till date. The work herein utilizes the simultaneous X-ray data from SXT and LAXPC units. The UVIT data contains the data from only from 1-2 filters and therefore this data does not seem to be useful for broadband spectral analysis. The observation details are reported in Table 1. The following sections include the instrument details of SXT \& LAXPC and the involved data analysis steps.

\subsection{SXT}

SXT is a focusing telescope capable of X-ray imaging and spectroscopy in the energy range $0.3-8.0 \mathrm{keV}$ with $2^{\prime}$ angular resolution and FOV of $\sim 40^{\prime}$ diameter $[18,19]$. The Level-1 SXT 
data for both the sources was observed in the photon counter (PC) mode and this data was first processed with sxtpipeline available in the SXT software (AS1SXTLevel2, version 1.4). The sxtpipeline calibrates the source events and extracts Level-2 cleaned event files for the individual orbits obtained for the given exposure time. The cleaned event files of all orbits were then merged into a single cleaned event file using by the sxtevtmerger $j 1$ tool developed by the instrumentation team to avoid the time-overlapped event files from the consecutive orbits. To extract the source spectrum from the processed Level-2 cleaned event files, XSELECT V2.4d package built-in HEAsoft was used with a source region as a circular region of 15 arcmin radius centred at the source position. However, the source region was determined by using the sxtEEFmaker tool and was checked for possible pile-up. No effect of pile-up was detected for these sources. The background extraction was carried out with a deep blank sky observation and the used background spectrum ("SkyBkg_comb_EL3p5_Cl_Rd16p0_v01.pha") was taken from the SXT website. The ARF was generated for faint sources by using sxtmkarf tool as recommended by the instrumentation team. The response file "sxt_pc_mat_g0to12.rmf", used was downloaded from the instrument's website. Various data reduction tools, background and the response files are available for download at the SXT webpage ${ }^{1}$. The source spectrum was then grouped using the grppha tool to ensure a minimum of 60 counts.

\subsection{LAXPC}

LAXPC is the X-ray proportional counter consists of three identical co-aligned proportional counter units providing with high time resolution $(\sim 10 \mu \mathrm{s})$ covering $3-80 \mathrm{keV}$ energy band $[20,21]$. The proportional counter units are named as LAXPC10, LAXPC20 and LAXPC30 with a total detector effective area of $\sim 6000 \mathrm{~cm}^{2}$ and $\sim 2000 \mathrm{~cm}^{2}$ each. To process the Level-1 LAXPC data, the laxpc_soft packages were used which is developed by the instrument team and available at the AstroSat Science Support Cell (ASSC) website ${ }^{2}$. The data reduction procedures involve the generation of event files, good time intervals (GTI files) to avoid Earth occultation and the South Atlantic Anomaly, and finally, the extraction of spectra for the source and the background. The final background spectrum was regenerated for a faint source by extracting the background with a GTI corresponding to the $50-80 \mathrm{keV}$ lightcurve [22]. The data of each LAXPC unit (LAXPC10, 20 and 30 ) is reduced separately and the data from the top layers (layer 1) from each unit is recommended especially for the faint sources. For this analysis, we have used the LAXPC data from LAXPC20 unit which provides better data statistics compared to LAXPC10 unit or LAXPC30 due to the continuous gain shift observed in LAXPC30 unit [21].

\section{Spectral Models and analysis}

The X-ray spectral studies of these sources performed in the previous works cited, have provided us with only a vague idea about their spectral nature. The observed XRT spectrum for 1ES 1741+196, in particular, has shown a hard power-law spectral index and is associated with large uncertainty in locating its synchrotron peak energy. In this work, we utilize the soft X-ray data from SXT jointly with the LAXPC20 data, a wide range X-ray spectrum extracted for the energy range

${ }^{1}$ https://www.tifr.res.in/ astrosat_sxt/index.html

${ }^{2} \mathrm{http}: / /$ astrosat-ssc.iucaa.in/?q=sxtData 
Table 1: Observation details of SXT \& LAXPC instruments aboard AstroSat

\begin{tabular}{ccccc}
\hline \hline Source & Observation ID & $\begin{array}{c}\text { Observation date } \\
(\text { yyyy-mm-dd) }\end{array}$ & $\begin{array}{c}\text { Exposure (SXT) } \\
(\mathrm{ks})\end{array}$ & State \\
\hline \hline \multirow{3}{*}{ 1ES 1741+196 } & AO5-163T01-9000002820 & $2019-03-27$ & 33.94 & S1 \\
& AO5-163T01-9000003010 & $2019-07-03$ & 33.12 & S2 \\
& AO5-163T01-9000003118 & $2019-08-22$ & 24.8 & S3 \\
\hline 1ES 2322-409 & AO9-147T01-9000003754 & $2020-07-03$ & 44.21 & \\
\hline \hline
\end{tabular}

of 0.3 - $10 \mathrm{keV}$. However, LAXPC data for 1ES 2322-409 was discarded due to poor data quality. We use a simple power-law (powerlaw) and log-parabola (logpar) models built in XSPEC in order to have a quantitative idea about the observed spectrum. The simple power-law gives a poor fit to the observed spectra. The functional form of log-parabola model is given as [5, 6],

$$
F(E)=K\left(\frac{E}{E_{0}}\right)^{-\left[\alpha+\beta \log \left(\frac{E}{E_{0}}\right)\right]}
$$

Where $\alpha$ and $\beta$ are the fit parameters representing spectral index at $E_{0}$ and the measurement of curvature respectively. $K$ is the normalization factor, whereas $E_{0}$ denotes pivot energy which is kept at $1 \mathrm{keV}$ for our analysis.

Spectral fittings for each observation are performed using XSPEC (Version 12.11.1) software package distributed with HEASoft and the best-fit parameters, $\alpha, \beta$ values are reported in Table 2. The peak energy $\mathrm{E}_{p}$ values in Table 2 are estimated using eplogpar model in-built in XSPEC. The correction due to galactic absorption due to neutral hydrogen is performed by using TBabs model available in XSPEC. The value of equivalent-hydrogen column density $\left(\mathrm{N}_{H}\right)$ fixed at $7.36 \times$ $10^{20} \mathrm{~cm}^{-2}$ for 1 ES $1741+196$ and $1.56 \times 10^{20} \mathrm{~cm}^{-2}$ for 1ES 2322-409 and are kept fixed throughout the analysis. These values are estimated by an online tool ${ }^{3}$ developed by the LAB survey group [23]. In addition, a 3\% systematic and a best-fit nominal gain offset of $0.03 \mathrm{keV}$ were added to SXT data by using the gain fit option with a gain slope of 1 as recommended by SXT instrument team. This significantly improves the fit statistics for the SXT spectrum. To determine the relative cross-calibration uncertainties between two instruments, a multiplicative constant factor is used.

We further consider the EDD model to investigate the spectral curvature and to attain deeper insight into the underlying physical conditions. EDD model is based on two-zone leptonic scenario where particles are considered to be accelerated at shock fronts. The model considers that the curvature is arising due to an energy-dependent escape timescale of the electrons from the acceleration sites that takes the form $t_{\mathrm{esc}} \propto E^{-\kappa}$, with $E$ as the electron energy. The steady state electron distribution $N_{c}$ responsible for the observed emission spectrum is derived as,

$$
N_{c}(E) \propto E^{-2} \exp \left[-\frac{\eta_{0}}{\kappa}\left(\frac{E}{E_{0}}\right)^{\kappa}\right]
$$

\footnotetext{
${ }^{3}$ https://heasarc.gsfc.nasa.gov/cgi-bin/Tools/w3nh/w3nh.pl
} 
Table 2: Best-fit log-parabola model parameters of SXT-LAXPC spectrum using TBabs*logpar model

\begin{tabular}{|c|c|c|c|c|c|c|c|c|}
\hline State & Energy & Constant $\dagger$ & $\alpha$ & $\beta$ & $\mathrm{E}_{p}(\mathrm{keV})$ & $\mathrm{F}_{0.5-2 \mathrm{keV}}$ & $\mathrm{F}_{2-10 \mathrm{keV}}$ & $\chi_{r e d}^{2}\left(\chi^{2} / \mathrm{dof}\right)$ \\
\hline 1ES 1741+196 & $0.3-10 \mathrm{keV}$ & & & & & & & \\
\hline $\mathrm{S} 1$ & - & $1.08_{-0.13}^{+0.13}$ & $1.75_{-0.05}^{+0.05}$ & $0.41_{-0.09}^{+0.10}$ & $2.07_{-0.20}^{+0.22}$ & $1.14_{-0.02}^{+0.01}$ & $1.54_{-0.03}^{+0.07}$ & $1.18(281.50 / 238)$ \\
\hline S2 & - & $1.17_{-0.48}^{+0.54}$ & $1.73_{-0.05}^{+0.06}$ & $0.45_{-0.12}^{+0.13}$ & $1.98_{-0.19}^{+0.21}$ & $1.07_{-0.01}^{+0.03}$ & $1.45_{-0.05}^{+0.14}$ & $1.09(240.58 / 219)$ \\
\hline S3 & - & $0.91_{-0.14}^{+0.17}$ & $2.01+0.06$ & $0.16_{-0.13}^{+0.14}$ & $0.98_{-0.38}^{+0.27}$ & $0.91_{-0.02}^{+0.01}$ & $1.10_{-0.06}^{+0.05}$ & $0.87(157.93 / 181)$ \\
\hline 1ES 2322-409 & $0.3-5 \mathrm{keV}$ & - & $2.31_{-0.05}^{+0.05}$ & $\begin{array}{l}0.32_{-0.19}^{+0.20} \\
\end{array}$ & $0.33_{-0.20}^{+0.27}$ & $0.56_{-0.01}^{+0.02}$ & $\begin{array}{c}0.32+0.07 \\
-0.04 \\
\end{array}$ & $1.13(154.39 / 136)$ \\
\hline
\end{tabular}

Note: The average flux (F) is in units of $10^{-11} \mathrm{erg} \mathrm{cm}^{-2} \mathrm{~s}^{-1} . \alpha$ is estimated at $1 \mathrm{keV}$. $\dagger$ The relative cross-normalization constant, and is fixed at 1.0 for SXT and kept free for LAXPC data while performing joint SXT-LAXPC spectral fit.

The errors are estimated within $90 \%$ confidence range based on the criterion used in XSPEC.
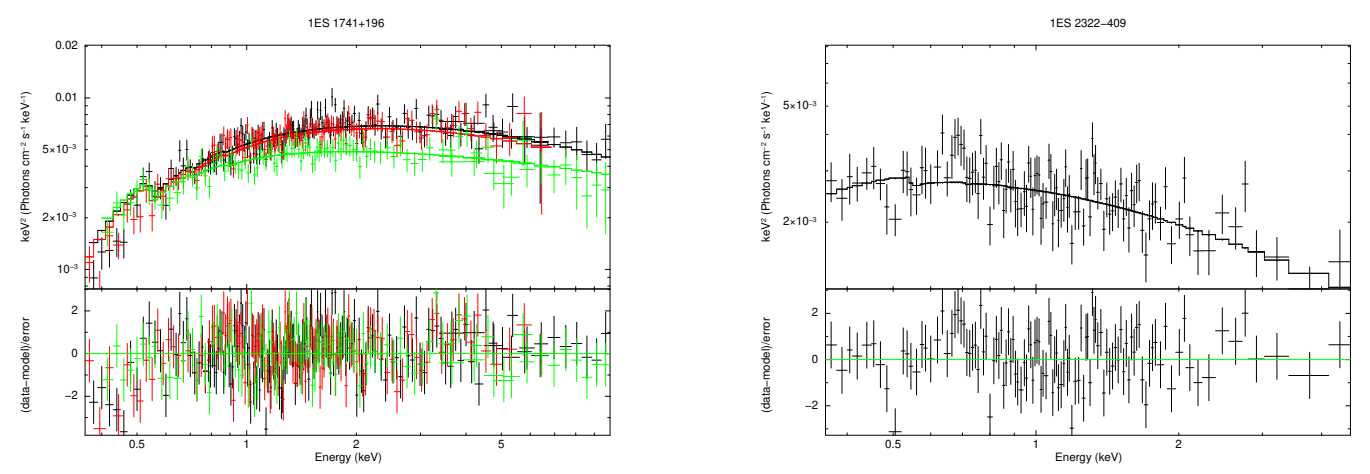

Figure 1: Figures show spectral fitting for combined SXT-LAXPC20 data on LHS (S1:black, S2: red and S3:green) for 1ES 1741+196 and SXT data for 1ES 2233-409 on RHS using log-parabola model

The observed synchrotron flux is then obtained by convolving $N_{c}(E)$ with the single particle emissivity function after accounting for relativistic effects. The model describes the observed synchrotron spectrum in terms of two parameters, $\psi$ and $\kappa$. The parameter $\kappa$ is associated with the electron diffusion timescale that decides the observed spectral curvature. The best-fit parameters using EDD model are reported in Table 3. The synchrotron peak energy values $\epsilon_{p}$ are estimated by using a relation derived between the peak, $\epsilon_{p}$ and the model parameters $\psi$ and $\kappa$ (Refer to Goswami et al. (2020) [8] for the detailed derivation).

\section{Results and Discussion}

In the first instance, we obtain a poorer fit of the simple power-law model, indicating the presence of intrinsic curvature in the X-ray spectrum for both sources. The log-parabola model in contrast, shows very good statistical fits to all three-epoch SXT-LAXPC spectra (S1, S2 \& S3) of 1ES 1741+196 and SXT spectrum of 1ES 2322-409 (refer to Table 2). As for 1ES 1741+196, very interestingly, the wide range SXT-LAXPC spectra are able to estimate the synchrotron peak well within the observation range at $\sim 1-2 \mathrm{keV}$. This leads to the first well-constrained determination 
Table 3: Best-fit EDD model parameters of joint SXT-LAXPC spectra

\begin{tabular}{|c|c|c|c|c|c|}
\hline State & Energy & $\psi$ & $\kappa$ & $\epsilon_{p}(\mathrm{keV})$ & $\chi_{\text {red }}^{2}\left(\chi^{2} / \mathrm{dof}\right)$ \\
\hline 1ES 1741+196 & $0.3-10 \mathrm{keV}$ & & & & \\
\hline S1 & - & $10.15_{-0.89}^{+0.92}$ & $0.82_{-0.05}^{+0.06}$ & $2.17_{-0.60}^{+0.92}$ & $0.99(236.34 / 238)$ \\
\hline S2 & - & $9.05_{-1.44}^{+1.56}$ & $0.78_{-0.06}^{+0.07}$ & $1.95_{-0.47}^{+0.67}$ & $1.06(232.75 / 219)$ \\
\hline S3 & - & $\begin{array}{l}4.78_{-0.99}^{+1.09} \\
\end{array}$ & $0.52_{-0.08}^{+0.09}$ & $0.86_{-0.19}^{+0.29}$ & $0.81(147.16 / 181)$ \\
\hline 1ES 2322-409 & $0.3-5 \mathrm{keV}$ & $4.32+0.30$ & $0.30_{-0.02}^{+0.02}$ & $9.53_{-0.35}^{+0.40} \times 10^{-3}$ & $1.15(157.37 / 136)$ \\
\hline
\end{tabular}

of the synchrotron peak of $1 E S 1741+196$ and the range of $\mathrm{E}_{p}$ further suggests these extreme peak values match the characteristics of an extreme HBL type source. Our analysis confirms that the HBL 1ES 1741+196 is an extreme HBL type. In addition, we see only a marginal variation in the indices, average flux and peak energies at different observational epochs. This may hint that the source does not show significant short term variability in X-rays. The spectral fit of 1ES 2322-409 shows a mild curvature in its SXT spectrum and the index value seems to be consistent with previously reported $\mathrm{XRT}$ index values. Further, $\mathrm{E}_{p}$ value is predicted at a lower energy around a few $\mathrm{eV}$, which is in accordance with a typical HBL behaviour.

The EDD model, on the other hand, provides an equally good statistical fit to these spectra (Refer to Table 3) indicating the curved X-ray spectral nature of these sources. However, we prefer the EDD model over a log-parabola, as this model is likely to provide a more detailed explanation to the observed curvature. EDD model interprets the curvature as a result of an energy-dependent electron diffusion and the curvature is determined in terms of the index of the energy-dependency factor $(\kappa)$. This factor $\kappa$ is further associated with the index of the MHD turbulent spectrum. Therefore, the extra information obtained from the EDD model is that the observed curved X-ray spectra may further be associated with Kolmogorov to Kraichnan type of diffusion during different flux states.

\section{Summary}

This work reports the preliminary spectral analysis results of the true simultaneous AstroSat data of the HBL sources 1ES 1741+196 and 1ES 2322-409. The X-ray emission features of these sources are poorly understood due to lack of sufficient data beyond XRT observation range. The major findings of this work are, 1) to establish the exact nature of the X-ray spectra of these sources at different epochs, and 2) to estimate the synchrotron spectral peak energy of 1ES 1741+196 within the wide range of X-ray energies. We estimated the synchrotron peak values and the best-fit values are well confined at high energies within the observation range. The study herein, therefore, reports the first well-constrained determination of the synchrotron peak of the source 1ES 1741+196 which is of EHBL class. However, in contrast to the previous studies where the X-ray spectrum is well fitted with a power-law model, our spectral analysis using SXT and LAXPC data reveals the curved $\mathrm{X}$-ray spectral nature of these sources. We interpreted the observed curvature as arising due to an energy-dependent electron diffusion, and the EDD model highlights a strong interlink between 
the curvature with the type of MHD turbulence. Finally, these extreme sources are potentially interesting in blazar studies as their unique properties challenge the standard emission mechanisms and acceleration theories. In this regard, a wide range X-ray and VHE observations are crucial to constrain the broadband SED and the emission parameters.

Acknowledgements: PG and RG acknowledge the financial support of ISRO under AstroSat archival Data utilization program. This publication uses data from the AstroSat mission of the Indian Space Research Organisation (ISRO), archived at the Indian Space Science Data Centre.

\section{References}

[1] Blandford R. D. \& Rees M. J., 1978, Phys. Scr.,17, 265

[2] Urry C. M., Padovani P., 1995, PASP, 107, 803

[3] Ghisellini G., Maraschi L., 1989, ApJ, 340, 181

[4] Padovani P., Giommi P., 1995, ApJ, 444, 567

[5] Massaro E., Perri M., Giommi P., Nesci R., 2004a, A\&A, 413, 489

[6] Massaro E., Perri M., Giommi P., Nesci R., Verrecchia F., 2004b, A\&A, 422, 103

[7] Goswami P., Sahayanathan S., Sinha A., Misra R., Gogoi R., 2018, MNRAS, 480, 2046

[8] Goswami, P., Sahayanathan, S., Sinha, A., Gogoi, R.2020, MNRAS, 499, 2094

[9] Costamante L., et al., 2001, A\&A, 371, 512

[10] Perlman, E. S., Stocke, J. T. et al., 1996, ApJS, 104, 251

[11] Heidt, J. et al., 1991, A\&A, 341, 683

[12] Donato, D. et al., 2005, A\&A, 433, 1163

[13] Ahnen, M. L. et al., 2017, MNRAS, 468, 1534

[14] Abeysekara, A. U. et al., 2016, MNRAS, 459, 2550

[15] Abdo, A. A. et al., 2009, ApJ, 707, 1310

[16] Abdalla H., et al., 2019, MNRAS, 482, 3011

[17] Agrawal P. C., 2006, Advances in Space Research, 38, 2989

[18] Singh K. P., Stewart, F. C., Chandra, S., et al. 2016, SPIE,9905, 1ES

[19] Singh K. P., et al., 2017, JAA, 38, 29

[20] Misra R., et al., 2017, ApJ, 835, 195

[21] Antia H. M., et al., 2017, ApJS, 231, 10

[22] Misra R., et al., 2021, JAA, arXiv:2102.06402

[23] Kalberla P. et al., 2005, A\&A,440, 775 